\section{Facharztprüfung zur Erlangung des Facharzttitels FMH für Gynäkologie und Geburtshilfe}

Aufgrund des Weiterbildungsprogrammes und des Beschlusses des Zentralvorstandes der FMH vom 3. Februar 1999 ist das Bestehen der Facharztprüfung für alle Kandidatinnen und Kandidaten, welche ihre Weiterbildung nach dem 31. Dezember 2000 abschliessen, Voraussetzung zur Erlangung des Facharzttitels FMH für Gynäkologie und Geburtshilfe. Näheres zu den Übergangsbestimmungen und Ausnahmen ist in der Schweizerischen Ärztezeitung Nr. 6 vom 7. Februar 2001 publiziert.
Anmeldung: Generalsekretär der SGGG, Prof. Dr. med. M. Litschgi, Abteilung Geburtshilfe/Gynäkologie, Kantonsspital, 8208 Schaffhausen, Tel. 052 63423 15, Fax 0526342398

Prüfungsgebühr: Fr. 1800.-. Ausserordentliche Mitglieder der SGGG bezahlen bereits mit ihren Jahresbeiträgen einen Teil des Examens im voraus. Der Vorstand bestimmt die Höhe dieses Betrages.

Anmeldefrist: 31. Juli 2001

Die Operationsberichte gemäss Anforderungskatalog müssen bis spätestens 15. Juli 2001 dem Vertreter der SGGG in der Titelkommission, Dr. med. H. Welti, 12, avenue de Valmont, Case postale 362, 1010 Lausanne 10, geschickt werden.

Die FMH-Zeugnisse und die Evaluationsprotokolle müssen bis 15. Juli 2001 an die Abteilung Weiterund Fortbildung, Verbindung der Schweizer Ärztinnen und Ärzte FMH, Elfenstrasse 18, 3000 Bern 16, gesandt werden.

\section{Examen de spécialiste pour l'obtention du titre FMH en gynécologie et obstétrique}

Conformément au programme de formation postgraduée et à la décision du Comité central de la FMH du 3 février 1999, la réussite de l'examen de spécialiste est une condition requise pour les candidats au titre FMH en gynécologie et obstétrique qui termineront leur formation postgraduée après le 31 décembre 2000. Pour de plus amples renseignements concernant les dispositions transitoires et les dérogations, veuillez vous référer à la publication du Bulletin des médecins suisses no 6, du 7 février 2001.
Inscription: Secrétariat de la SSG0, Prof. Dr. med. M. Litschgi, Département de gynécologie et obstétrique, Hôpital cantonal, 8208 Schaffhouse, tél. 052 63423 15, fax 0526342398

Taxe d'examen: Fr. 1800.-. Les membres associés de la SSGO paient déjà à l'avance une partie de cette taxe avec leurs cotisations annuelles. Le Comité détermine la hauteur de ce montant.

Délai d'inscription: le 31 juillet 2001

Les protocoles opératoires selon le catalogue des exigences doivent être envoyés jusqu'au 15 juillet 2001 au plus tard au délégué de la SSGO à la Commission des titres, le Dr H. Welti, 12, avenue de Valmont, Case postale 362, 1010 Lausanne 10.

Les certificats FMH et les protocoles d'évaluation doivent être envoyés jusqu'au 15 juillet 2001 au Département de la formation postgraduée et continue, Fédération des médecins suisses FMH, Elfenstrasse 18, 3000 Berne 16. 